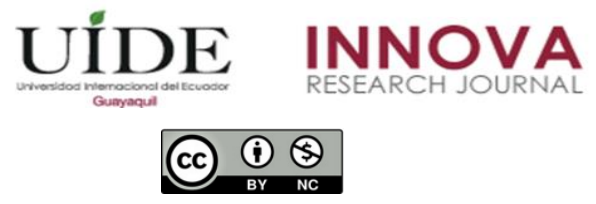

INNOVA Research Journal, ISSN 2477-9024

(Enero - Abril 2021). Vol. 6, No.1 pp. 47-60

DOI: https://doi.org/10.33890/innova.v6.n1.2021.1433

URL: http://revistas.uide.edu.ec/index.php/innova/index

Correo: innova@uide.edu.ec

\title{
Patrones matemáticos en los niveles de Inicial y Preparatoria: Análisis del currículo
}

\section{Preschool and Kindergarten Mathematical Patterns: Curriculum Analysis}

Gina Bojorque Iñegues

(iD https://orcid.org/0000-0002-5223-2829

Neli Gonzales Prado

(iD) https://orcid.org/0000-0003-3443-8468

Universidad de Cuenca, Ecuador

Autor para correspondencia: gina.bojorque@ucuenca.edu.ec; neli.gonzales@ucuenca.edu.ec Fecha de recepción: 21 de mayo de 2020 - Fecha de aceptación: 07 de septiembre de 2020

\section{Resumen}

El objetivo del presente estudio fue analizar el currículo ecuatoriano para subnivel Inicial II y Preparatoria, con un enfoque en patrones matemáticos, a la luz de la literatura internacional. La metodología empleada fue un análisis documental de dichos currículos enfocado en tres componentes, a decir, qué, cuándo y cómo enseñar. Los resultados del estudio demuestran que los currículos ecuatorianos para los niveles analizados incluyen, en general, patrones matemáticos simples de repetición, dejando menos evidente la presencia de patrones más complejos como son los patrones de crecimiento. Se encontró además que en los currículos no se incluye una secuencia clara a seguir para el trabajo con patrones, y que, si bien las recomendaciones metodológicas contempladas en los documentos curriculares sugieren diversas tareas para trabajar patrones, no se incluye la tarea de identificación de la unidad del patrón, la cual es fundamental para el desarrollo del pensamiento matemático del niño. De manera general, se concluye que, estos hallazgos apuntan a la necesidad de revisar el currículo nacional con miras a mejorar las competencias, en patrones en particular y en matemáticas en general, de los niños ecuatorianos desde los primeros años de escolaridad.

Palabras claves: currículo; patrones matemáticos; subnivel Inicial II; Preparatoria; análisis.

\begin{abstract}
The aim of the present study was to analyze the preschool and kindergarten Ecuadorian curriculum, with a focus on mathematical patterns, in light of the international literature. The method used was a documentary analysis of these curriculums focused on three components, namely what, when and how to teach. The results of the study demonstrate that the Ecuadorian curricula for the analyzed levels, generally include simple mathematical repeating patterns, with more complex patterns such as growing patterns being less evident. It was also found that the curriculums do not include a clear sequence to work with patterns, and that, although the methodological recommendations contemplated in the curricular documents suggest various tasks for working with
\end{abstract}


patterns, the identifying the unity of the pattern task, which is fundamental for the development of the child's mathematical thinking, is not included. In general, we concluded that these findings point to the need to review the national curriculum in view of improving Ecuadorian children's patterning competencies in particular, and mathematical competencies in general, from the first years of schooling.

Keywords: curriculum; mathematical patterns; Preschool; Kindergarten; analysis.

\section{Introducción}

El currículo es un esbozo de los requisitos de enseñanza y aprendizaje tanto del contenido como del desempeño y, por tanto, guía las experiencias de aprendizaje en las escuelas (Schmidt, McKnight, Valverde, Houang, y Wiley, 1997). Los estudios del currículo escolar, consecuentemente, son valiosos ya que permiten informar sobre las expectativas, los procesos y los resultados de las experiencias escolares de los estudiantes (Kulm y Li, 2009). Dichos estudios pueden realizarse a nivel del currículo en general o centrarse en determinadas áreas del mismo. Particularmente, en el área de matemáticas, Kulm y Li (2009) señalan que los esfuerzos realizados para alcanzar la excelencia en la educación matemática han llevado a revisiones y cambios continuos en el currículo matemático en varios sistemas educativos a nivel mundial. Por otro lado, varios autores coinciden en señalar que las competencias matemáticas que los niños desarrollan a temprana edad son los predictores más determinantes de su desempeño escolar futuro (por ejemplo, Duncan et al., 2007). Específicamente, los patrones matemáticos son un componente importante de la competencia matemática temprana ya que sustentan todas las ideas matemáticas (Björklund y Pramling, 2014; Nguyen et al., 2016; Rittle-Johnson, Fyfe, Hofer, y Farran, 2017). Más aún, el desarrollo de patrones matemáticos a temprana edad ha demostrado ser un potente predictor del desempeño matemático posterior (Bailey et al., 2016; Rittle-Johnson, Zippert, y Boice, 2019), por ello, un análisis del currículo nacional enfocado en patrones matemáticos en los primeros años de escolaridad, es fundamental ya que permitirá determinar si se está bridando a los niños pequeños las experiencias educativas necesarias que sirvan de base para que alcancen un desempeño matemático óptimo durante toda la escolaridad.

En el contexto ecuatoriano, aunque los patrones matemáticos están incluidos en los currículos de matemáticas para subnivel Inicial II y Preparatoria, desafortunadamente, hasta donde se conoce, no se han reportado estudios que evalúen si las destrezas relacionadas con patrones matemáticos estipuladas en los currículos son suficientes y adecuadas para los niños que asisten a dichos niveles educativos. Por ello, el objetivo de este estudio es analizar el currículo ecuatoriano de subnivel Inicial II y de Preparatoria, con un enfoque en patrones matemáticos, a la luz de la literatura internacional. De acuerdo al sistema educativo ecuatoriano, a la edad de cuatro años los niños asisten a subnivel Inicial II, mientras que, a la edad de cinco años, asisten a Preparatoria (primer año de educación básica). Ambos niveles educativos están regulados por el Ministerio de Educación y siguen un currículo oficial.

Para alcanzar el objetivo planteado, en primer lugar, se presentará una síntesis acerca de lo expuesto en la literatura internacional sobre patrones matemáticos. A continuación, se describirán las destrezas y actividades relacionadas con patrones matemáticos, propuestas en los 
documentos curriculares ecuatorianos para el subnivel Inicial II y Preparatoria. Finalmente, se realizará un análisis de lo prescrito en estos documentos, a la luz de la literatura internacional.

\section{Marco teórico}

\section{Patrones matemáticos}

Varios estudios internacionales han demostrado que las destrezas matemáticas que desarrollan los niños a temprana edad son predictores de su desempeño matemático a lo largo de la escuela (Aunio y Niemivirta, 2010; Duncan et al., 2007; Jordan, Kaplan, Ramineni, y Locuniak, 2009). Es decir, los niños pequeños que desarrollan destrezas matemáticas sólidas a temprana edad se desempeñan mucho mejor en toda su escolaridad en comparación con los niños que no adquieren dichas destrezas (Jordan, Kaplan, Ramineni, y Locuniak, 2009). Sin embargo, los estudios sobre la matemática temprana han sido cuestionados por su enfoque casi exclusivo en el desarrollo numérico, dejando de lado otras habilidades importantes como son los patrones matemáticos (Starkey, Klein, y Wakeley, 2004; Verschaffel, Torbeyns, y De Smedt, 2017). En este sentido, Clements y Sarama (2014) señalan que "el mundo matemático de los niños pequeños es mucho más rico" (p.537) y los patrones matemáticos juegan un papel importante. De hecho, diferentes estudios han demostrado que las habilidades para trabajar patrones matemáticos a temprana edad están fuertemente relacionadas con la adquisición de habilidades matemáticas generales (Lüken, Peter-Koop, y Kollhoff, 2014; Rittle-Johnson et al., 2017, 2019). Aunque no es fácil dar una definición de patrón matemático, Mulligan y Mitchelmore (2009) señalan que, al hablar de patrones matemáticos, es necesario distinguir entre un patrón, es decir, una entidad ordenada (opuesta a un arreglo aleatorio), y una estructura de patrón, es decir, la organización o regla que subyace al patrón. De acuerdo con estos autores, los patrones y estructuras, comprenden dos componentes: uno cognitivo (es decir, conocimiento de la estructura) y uno meta-cognitivo (es decir, tendencia a buscar patrones y analizarlos).

De acuerdo a Papic y Mulligan (2007), existen varios tipos de patrones que se pueden trabajar con los niños pequeños, siendo los más comunes los patrones de repetición, los patrones de crecimiento y los patrones de estructura espacial. Los patrones de repetición son patrones lineales que tienen una unidad que se repite indefinidamente (por ejemplo, círculo-triángulocírculo-triángulo-círculo-triángulo). Los patrones de crecimiento son patrones lineales que presentan una "unidad de patrón" que aumenta o disminuye sistemáticamente (por ejemplo, rojoazul-rojo-azul-azul- rojo-azul-azul-azul). Finalmente, los patrones de estructura espacial son invariantes y describen la organización de elementos individuales (por ejemplo, triángulo de puntos). Por otro lado, existen diversas tareas empleadas para trabajar el desarrollo de patrones matemáticos, entre estas tareas se encuentran las siguientes: (1) copiar, es decir, reproducir un patrón dado; (2) interpolar, hace referencia a completar elementos faltantes del patrón; (3) extender, es decir, continuar un patrón dado; (4) generalizar, se refiere a realizar el mismo patrón con diferentes materiales; (5) identificar, hace referencia a reproducir el patrón de memoria luego de haber identificado la unidad de patrón; y, (6) crear, quiere decir, inventar un patrón (Wijns, Torbeyns, De Smedt, y Verschaffel, 2019). 
Diversos autores señalan que la realización de actividades con patrones matemáticos a temprana edad es factible y adecuada, ya que se ha observado que varios niños crean espontáneamente patrones matemáticos durante el juego libre (Piccolo y Test, 2010; Seo y Ginsburg, 2004). Estudios previos han documentado que los niños progresan en sus habilidades para trabajar patrones matemáticos desde el nivel prescolar hasta el jardín infantil (Clements y Sarama, 2014; Lüken, 2018; Rittle-Johnson, Fyfe, Loehr, y Miller, 2015; Rittle-Johnson B. , Fyfe, McLean, y McEldoon, 2013). Dichas habilidades van desde la capacidad de interpolar, copiar y extender patrones de repetición simples (es decir, patrones AB) a los cuatro años, pasando por la capacidad de extender patrones de repetición un poco más complejos (por ejemplo, ABB o ABC) a los cinco años, hasta la capacidad de identificar la unidad más pequeña de un patrón (por ejemplo, en un patrón $\mathrm{ABB} A B \mathrm{BABB}$, el niño identifica la unidad central del patrón como $\mathrm{ABB}$ ) a la edad de seis años (Clements y Sarama, 2014).

Según Wijns et al. (2019) un número cada vez mayor de países ha incluido patrones matemáticos en su currículo de matemáticas inicial. Sin embargo, varios autores señalan que, en la mayoría de currículos de preescolar, el enfoque en patrones matemáticos se centra en patrones de repetición simples (por ejemplo, $\mathrm{AB}-\mathrm{ABB}$ ), por lo que se debe prestar más atención a patrones más complejos como patrones de crecimiento (por ejemplo, AB-ABB o 1-3-5) (Kidd et al., 2013), pues este tipo de patrones permite desarrollar un "pensamiento funcional", según el cual la actividad de patrones se basa en una comprensión más profunda y general de la estructura o regla subyacente a toda la secuencia, lo cual es mucho más valioso (Papic y Mulligan, 2007; Rittle-Johnson et al., 2013).

\section{Documentos curriculares}

De acuerdo al Ministerio de Educación (2016), en el currículo ecuatoriano se evidencian en cierto grado las intenciones educativas del país, se presentan orientaciones para hacer realidad dichas intenciones y se dan a conocer lineamientos para comprobar si efectivamente se han alcanzado. De esta manera el currículo se encarga de informar a los docentes sobre lo que se quiere conseguir y los orienta sobre cómo hacerlo, por otro lado, es un referente para la rendición de cuentas del sistema educativo y para la evaluación de dicho sistema.

Los documentos curriculares analizados en el presente estudio, son aquellos que están disponibles en la página web del Ministerio de Educación del Ecuador. En relación al subnivel Inicial II, los documentos analizados incluyen, el currículo de educación inicial (Ministerio de Educación, 2014) y la guía metodológica para la implementación del currículo de educación inicial (Ministerio de Educación, 2015). Por su parte, los documentos curriculares de Preparatoria comprenden, el currículo de los niveles de educación obligatoria (Ministerio de Educación, 2016), el cuaderno de trabajo de relaciones lógico matemáticas (Ministerio de Educación, 2018), y la guía del docente para Preparatoria (Ministerio de Educación, 2017).

\section{Currículo ecuatoriano para el subnivel Inicial II (niños de 4 a 5 años): Patrones matemáticos}

En el subnivel Inicial II, el currículo nacional plantea tres ejes de desarrollo y aprendizaje, a decir, desarrollo personal y social, descubrimiento natural y cultural, y expresión y 
comunicación, cada uno de ellos abarca diferentes ámbitos. Dentro del eje de desarrollo denominado descubrimiento natural y cultural, se encuentra el ámbito de relaciones lógicomatemáticas, en el cual se trabajan destrezas relacionadas con nuestro foco de análisis que son los patrones matemáticos.

Uno de los objetivos de aprendizaje de este nivel, en el ámbito de relaciones lógicomatemáticas, es "comprender nociones básicas de cantidad facilitando el desarrollo de habilidades del pensamiento para la solución de problemas sencillos" (Ministerio de Educación, 2014, p. 37). Para alcanzar este objetivo, se plantea una sola destreza relacionada con patrones matemáticos, a decir, "continuar y reproducir patrones simples con objetos concretos y representaciones gráficas" (Ministerio de Educación, 2014, p. 37). Por otro lado, la guía metodológica para la implementación del currículo de educación inicial sugiere, en la página 28, que, en el rincón de juegos tranquilos, se incluyan materiales para ensartar y seguir o crear patrones (Ministerio de Educación, 2015). Dicha guía no realiza ninguna alusión adicional en lo que respecta a patrones matemáticos.

\section{Currículo ecuatoriano para Preparatoria (niños de 5 a 6 años): Patrones matemáticos}

El Currículo de los niveles de educación obligatoria, que incluye el nivel de Preparatoria presenta los contenidos básicos determinados por áreas de estudio y subniveles educativos evidenciados en las destrezas con criterios de desempeño. Dichos contenidos se enfocan en el reconocimiento de aprendizajes básicos imprescindibles (es decir, aquellos aprendizajes que son obligatorios, pues, si no se alcanzan en el nivel esperado, son difíciles de desarrollar en los años siguientes) y aprendizajes básicos deseables (es decir, aquellos aprendizajes que pueden alcanzarse con relativa facilidad en los siguientes años) (Ministerio de Educación, 2016).

En Preparatoria, el currículo nacional plantea tres destrezas relacionadas con patrones matemáticos, las cuales se contemplan dentro del bloque Álgebra y Funciones. La primera destreza corresponde a un básico imprescindible en tanto que las dos últimas son básicos deseables. Dichas destrezas según el Ministerio de Educación (2016) son:

Describir y reproducir patrones con objetos del entorno por color, forma, tamaño, longitud o con siluetas de figuras geométricas, sonidos y movimientos. Describir y reproducir patrones con cuerpos geométricos. Y, describir y construir patrones sencillos agrupando cantidades de hasta diez elementos. (p. 313)

Con respecto al cuaderno de trabajo para el estudiante (en el área de relaciones lógicomatemáticas), existen únicamente seis actividades que plantean el empleo de patrones matemáticos. Estas actividades se centran en trabajar ejercicios de continuar patones de repetición (cuatro ejercicios) o de continuar patrones de crecimiento (dos ejercicios), atendiendo a uno o dos de los siguientes criterios: color, forma, cantidad, tamaño, movimiento y sonido (Ministerio de Educación, 2018).

En relación a la guía para el docente de Preparatoria, se presenta una definición de patrón y se dan sugerencias metodológicas para trabajar los patrones y para desarrollar las actividades planteadas en el cuaderno de trabajo para el estudiante. En esta guía, se define a un patrón como 
"una secuencia en que cada elemento, ocupa un lugar que se le ha asignado según una regla determinada con anticipación" (Ministerio de Educación, 2017, p. 86). Una vez presentada la definición se presentan seis sugerencias metodológicas para el trabajo con patrones, estas sugerencias - según el Ministerio de Educación (2017) - son:

(1) Trabajar patrones de dos y más elementos a través de sonidos, aplausos, posición de los cuerpos, parados, sentados, acostados, manos arriba, abajo, etc.; (2) ordenar a los niños bajo algún patrón establecido: hombre-mujer, mujer-mujer, hombre-hombre, etc., (3) repartir dos, tres o cuatro figuras diferentes, de manera que a cada niño le toque una sola figura; luego, que las ordenen formando un patrón. Cada niño tendrá la oportunidad de crear un patrón; (4) formar patrones con lápices de colores, agrupar al curso de a diez o menos niños; a cada grupo le toca un color, para luego formar patrones diversos; (5) escribir patrones en la pizarra; los niños irán dictando; y, (6) crear patrones en hojas de trabajo y compartirlos con los compañeros. Colocar una cinta numérica en el suelo, jugar con los niños a avanzar, de dos en dos, de tres en tres y reconocer los números. (pp. 85-86)

Finalmente, el documento presenta algunas sugerencias para trabajar antes, durante y después de las seis actividades planteadas en el cuaderno de trabajo para el estudiante.

\section{Resultados}

\section{Análisis de los currículos ecuatorianos de subnivel Inicial II y Preparatoria en relación a patrones matemáticos}

En esta sección se realizará un análisis de lo prescrito en los documentos curriculares, de los subniveles Inicial II y Preparatoria, sobre patrones matemáticos en relación con la literatura internacional existente. Dicho análisis se realizará en base a tres de los criterios considerados básicos en un análisis del currículo, estos criterios son: qué enseñar (contenidos/destrezas); cuándo enseñar (secuencia); y cómo enseñar (metodología) (Bojorque et al, 2015; Baroody, 2004). El qué enseñar se refiere a los contenidos planteados en el currículo. Según Stein, Remillard y Smith (2007) si algún contenido no está mencionado en el currículo, es muy probable que los docentes no lo trabajen y por lo tanto los niños no lo van a aprender. Por lo tanto, en el proceso de desarrollo del currículo se debe prestar especial atención a la selección de contenidos a ser trabajados en cada área y nivel. El cuándo enseñar se refiere a la secuencia en la cual se enseñan los diferentes contenidos o destrezas planteados en el currículo. Dicha secuencia debe ser cuidadosamente diseñada para permitir que los niños vayan construyendo paulatinamente una red de conocimientos de acuerdo a su nivel de edad, la cual servirá de cimiento para desarrollar un conocimiento más avanzado (Fyfe, Rittle-Johnson, y DeCaro, 2012; Griffin, 2005). En este sentido, Baroody, Clements y Sarama (2019) señalaron que la instrucción debe guiarse por las trayectorias de aprendizaje (véase, Clements y Sarama, 2014 para detalle de las trayectorias de aprendizaje en matemáticas) de los niños, dichas trayectorias incluyen niveles de desarrollo basados en la teórica y probados empíricamente, proporcionan una dirección clara

y específica para identificar los niveles de desarrollo actuales de los niños y por lo tanto permiten diseñar la instrucción para ayudarlos a alcanzar el siguiente nivel, independientemente del contexto y de la estrategia de enseñanza empleada. Finalmente, el cómo enseñar hace referencia 
a las estrategias de enseñanza y aprendizaje implementadas durante el proceso educativo. Baroody (2004) aclaró que es importante proporcionar a los educadores indicios claros y válidos sobre cómo enseñar a los niños pequeños, en general, y cómo ayudarlos a aprender habilidades específicas, conceptos y competencias de indagación en particular. En este sentido, Epsteing (2007) manifestó que es necesario que los profesores cuenten con una compilación de estrategias efectivas de enseñanza y que sepan cuándo emplear una determinada estrategia acorde a la destreza a ser desarrollada por sus estudiantes.

\section{¿Qué enseñar?}

En relación al qué enseñar, es decir, a las destrezas propuestas en el currículo nacional, se indicó anteriormente que, en el subnivel Inicial II, se plantea una sola destreza a ser desarrollada por los niños de cuatro años, dicha destreza consiste en "continuar y reproducir patrones simples con objetos concretos y representaciones gráficas” (Ministerio de Educación, 2014, p. 37). En dicha destreza, se pone de manifiesto en primer lugar, que el niño sea capaz de continuar y copiar un patrón dado y, en segundo lugar, que trabaje con patrones simples. Hay que resaltar que el currículo nacional incluye patrones matemáticos simples para trabajar con niños de cuatro años, siendo estos los primeros patrones con los que interactúan los niños pequeños (RittleJohnson et al., 2015), sin embargo, varios estudios (véase, Lüken, 2018; Rittle-Johnson, et al., 2015; Clements y Sarama, 2014) han reportado que los niños de cuatro años de edad están en capacidad de aprender patrones matemáticos un tanto más complejos. Por ejemplo, en un estudio realizado en una muestra de jardines de infantes en Alemania, Lüken (2018) encontró que los niños de cuatro años eran capaces no solo de copiar patrones sino también de interpolar y extender tanto patrones simples del tipo $\mathrm{AB}$ como patrones un poco más complejos del tipo $\mathrm{ABC}$ y AAB. Más aún en dicho estudio, los niños fueron capaces de realizar tareas de generalizar patrones, es decir, crear un patrón similar a un modelo dado, pero con figuras diferentes.

Por otro lado, como se mencionó anteriormente, en el currículo de Preparatoria se plantea una destreza imprescindible y dos destrezas deseables, relacionadas con patrones matemáticos, las cuales se centran exclusivamente en describir y reproducir patrones con objetos del entorno y cuerpos geométricos y, en construir patrones sencillos. Nuevamente, se resalta lo valioso de incluir este tipo de patrones en el currículo nacional de Preparatoria, no obstante, la literatura internacional señala que los niños de cinco años de edad son capaces no solamente de describir, reproducir y construir patrones sencillos sino también de realizar una variedad de actividades con patrones un poco más complejo (Coburn et al, 1993; Clements \& Sarama, 2014; Economopoulos, 1998). Por ejemplo, Coburn et al. (1993) señalaron que, en el jardín infantil, cuando los niños tienen cinco años de edad, el currículo debe incluir actividades que les permita enfocarse en la regularidad y la repetición de patrones; en aspectos relacionados con el movimiento, color, sonido, posición, y cantidad; y estar involucrados en reconocer, describir, extender, generalizar y crear patrones.

Con respecto al el tipo de patrones matemáticos (es decir, patrones de repetición -AB$\mathrm{AB}-\mathrm{AB}-\mathrm{o}$ patrones de crecimiento $-\mathrm{AB}-\mathrm{ABB}-\mathrm{ABBB}-$ ), los documentos curriculares del subnivel Inicial II no especifican si el trabajo se debe realizar con patrones de repetición o con patrones de crecimiento. En el caso del currículo de Preparatoria tampoco se especifica el tipo de patrón a ser trabajado, sin embargo, en la guía para el docente de este nivel, se hacen 
recomendaciones solamente para el trabajo con patrones de repetición sencillos, mientras que en el cuaderno de trabajo para el estudiante se proponen cuatro actividades con patrones de repetición y dos con patrones de crecimiento. Si bien, se evidencia en el cuaderno de trabajo para el estudiante de Preparatoria la inclusión de dos actividades con patrones de crecimiento (lo cual demuestra que los autores del cuaderno de trabajo consideraron importante incluir este tipo de patrones), es necesario prestar más atención a este tipo de patrones ya que, como se mencionó anteriormente, el trabajo con patrones de crecimiento (por ejemplo, círculo-cuadrado, círculocuadrado-cuadrado) es más enriquecedor pues, permite que el niño desarrolle una comprensión más profunda y general de la regla subyacente a toda la secuencia (Kidd et al., 2013). Así mismo, Coburn et al. (1993), señalaron que para entender el concepto de patrón los niños deben reconocer que los patrones son predecibles y que están compuestos por una unidad que se repite; además, que algunos patrones repiten la unidad básica (por ejemplo, rojo-rojo-azul-rojo-rojoazul) mientras que, otros patrones parecen crecer (por ejemplo, aplauso-salto-aplauso-aplausosalto-aplauso-aplauso-aplauso-salto).

Finalmente, al analizar el cuaderno de trabajo para el estudiante de Preparatoria se evidenció que, en algunos casos, las destrezas presentadas no se corresponden con la actividad propuesta. Por ejemplo, en la página 27, la destreza propuesta es "escribir y reproducir patrones con objetos del entorno por su forma y color", sin embargo, el ejercicio requiere prestar atención al color y la cantidad. En la página 30, la destreza es "describir y reproducir patrones con objetos del entorno por su longitud", no obstante, en el ejercicio se pide ordenar objetos por el tamaño, mas no por su longitud. Por otro lado, al comparar las destrezas planteadas en las actividades del cuaderno de trabajo con las destrezas planteadas en la guía docente para el desarrollo de esas actividades, se evidencia que dichas destrezas no coinciden. Por ejemplo, la destreza con criterio de desempeño presentada en la página 27 del cuaderno de trabajo es "describir y reproducir patrones con objetos del entorno por su forma y color"; sin embargo, en la guía del docente, la destreza para esa actividad es "describir y construir patrones sencillos agrupando cantidades de hasta diez elementos”. La destreza planteada para las demás actividades en la guía del docente es "describir y reproducir patrones con objetos del entorno por color, forma y cantidad" pero la misma no siempre guarda relación ni con las destrezas ni con actividades del cuaderno de trabajo. Si bien es importante que en los documentos curriculares se planteen destrezas claras que guíen el trabajo docente, es igual de importante que exista coherencia en el planteamiento de dichas destrezas tanto dentro de un mismo documento como entre los documentos relacionados, con el fin de que los lineamientos sean claros para el docente y no haya lugar a confusión.

\section{¿Cuándo enseñar?}

Con respecto al cuándo enseñar, o sea, la secuencia a seguir para la enseñanza de patrones matemáticos, se encontró que, en ninguno de los dos niveles analizados el currículo especifica una secuencia para dicha enseñanza; sin embargo, en ambos niveles, las destrezas que hacen referencia a patrones matemáticos forman parte de las primeras destrezas presentadas en los currículos. La inclusión de patrones matemáticos dentro las primeras destrezas a ser trabajadas por los niños pequeños es indispensable ya que los patrones matemáticos sustentan todas las ideas matemáticas y permiten conectar dicho aprendizaje con el aprendizaje matemático posterior; por ejemplo, cuando los niños empiecen más tarde a contar de dos en dos (es decir, 2, $4,6,8)$ podrán notar rápidamente que existe un patrón en dichos números (Björklund y

Esta obra se comparte bajo la licencia Creative Common Atribución-No Comercial 4.0 International (CC BY-NC 4.0) Revista de la Universidad Internacional del Ecuador. URL: https://www.uide.edu.ec/ 
Pramling, 2014; Nguyen et al., 2016; Rittle-Johnson et al., 2017). No obstante, los patrones matemáticos deberían ser trabajados a lo largo de todo el año lectivo, no solo en diferentes temas matemáticos sino también en relación con otras áreas de aprendizaje, como lengua, arte, música, o ciencias, las cuales involucran el uso de patrones. Por ejemplo, la música implica crear patrones de sonido como: aplauso-aplauso-aplauso-pausa-aplauso-aplauso, lo cual a su vez está relacionado con el concepto cardinal de tres y dos (es decir, tres aplausos, pausa y luego dos aplausos más).

En el cuaderno de trabajo para el estudiante de Preparatoria, se presentan seis actividades para trabajar patrones matemáticos mismas que no evidencian seguir una secuencia clara y ordenada, dichas actividades son: describir y reproducir patrones por color y cantidad (actividad 1); por su forma y color (actividad 2); por su forma, color y tamaño (actividad 3); por su tamaño (actividad 4); describir y construir patrones sencillos agrupando cantidades de hasta 10 elementos (actividad 5); y, finalmente, describir y reproducir patrones con objetos del entorno por movimientos y sonidos (actividad 6). Al respecto, y como se mencionó anteriormente, los niños siguen una trayectoria de aprendizaje natural por ello, es importante que en el currículo se establezcan pautas claras sobre la secuencia lógica a seguir en el aprendizaje de patrones matemáticos, las cuales permitan a los docentes en primer lugar, identificar en dónde se encuentra cada niño en la trayectoria de aprendizaje, en segundo lugar, determinar la siguiente destreza a ser desarrollada, y finalmente, planificar actividades para ayudarlos a alcanzar dicha destreza. Un ejemplo de la trayectoria de aprendizaje de patrones matemáticos descrita por Clements y Sarama (2014), la cual va de lo más sencillo a lo más complejo, es la siguiente: reconocer un patrón simple (identificar en una camiseta un patrón de rayas: rojo-azul-rojo-azul); completar el elemento que le falta a un patrón (identifica y completa el objeto que falta: ABAB_BAB); duplicar un patrón (dado el patrón $\mathrm{AB}-\mathrm{AB}-\mathrm{AB}$, puede hacer su patrón $\mathrm{AB}-\mathrm{AB}$ $\mathrm{AB}$ ); extender un patrón (presentado el patrón $\mathrm{ABB}-\mathrm{ABB}-\mathrm{ABB}$, añade $\mathrm{ABB}$ al final); e identificar la unidad de patrón (dado el patrón $\mathrm{ABB}-\mathrm{ABB}-\mathrm{ABB}$, identifica $\mathrm{ABB}$ como la unidad central del patrón).

\section{¿Cómo enseñar?}

Finalmente, en cuanto al cómo enseñar, es decir, a la metodología sugerida para la enseñanza de patrones matemáticos, en los currículos del subnivel Inicial II y de Preparatoria se señala, entre otras recomendaciones, que el ambiente de aprendizaje deberá ser cálido y afectivo, con un tipo de educación activa, social y guiada; se rescata la importancia de promover aprendizajes significativos y sobre todo el empleo de estrategias de enseñanza lúdicas que provoquen regocijo en los niños, con miras a que su inicio en la etapa de escolarización resulte placentero y motivante (Ministerio de Educación, 2016; 2014). No cabe duda que estas recomendaciones son valiosas y esenciales para el trabajo con niños pequeños. Específicamente, en lo que respecta al desarrollo de actividades lúdicas, Baroody et al. (2019) consideran que el juego estructurado, elegido cuidadosamente en base a los intereses, las necesidades y el nivel de desarrollo de un niño pequeño, es un medio de desarrollo apropiado (agradable y educativo, desafiante pero alcanzable) para alcanzar objetivos académicos propuestos.

De manera puntual, en lo relacionado al trabajo con patrones matemáticos, en los documentos del subnivel Inicial II no se realizan recomendaciones, no así, en la guía para el 
docente de Preparatoria, en la cual se presentan varias sugerencias para el trabajo con patrones (las cuales fueron descritas anteriormente), entre ellas, trabajar patrones a través de sonidos, aplausos, movimientos del cuerpo, con el empleo de objetos del aula, y con figuras y colores. Es importante rescatar el hecho de que el currículo nacional recomiende el trabajo con patrones matemáticos presentados de modo visual (por ejemplo, figuras y colores), y de modo auditivo (por ejemplo, sonidos y aplausos); sin embargo, siguiendo a Lüken (2012), habría que incluir también actividades con patrones matemáticos presentados de modo táctil (por ejemplo, con los ojos vendados/cerrados formar un patrón con figuras reconocidas táctilmente).

En los documentos curriculares de Preparatoria también se recomienda trabajar actividades que permitan al niño crear (es decir, elaborar su propio patrón), copiar (es decir, reproducir un mismo patrón) y completar (es decir, continuar un patrón dado) patrones matemáticos, lo cual da cuenta de que se reconocen diferentes tipos de tareas para trabajar patrones con niños pequeños; no obstante, a esta edad también se deberían trabajar tareas que incluyan interpolar (es decir, completar elementos faltantes dentro del patrón), generalizar (es decir, realizar el mismo patrón con diferentes materiales), e identificar (es decir, reproducir el patrón de memoria luego de haber identificado la unidad de patrón) patrones matemáticos. En relación a la última tarea, identificar la unidad del patrón, Lüken (2018), recomienda que los profesores enseñen de manera explícita a los niños pequeños a identificar la estructura de los patrones, es decir, ayudarlos a "ver" cuál es la unidad mínima que constituye un patrón, dicho aprendizaje, según Economopoulos (1998), es un paso crucial en el desarrollo matemático de los niños.

\section{Conclusiones}

El objetivo de este estudio fue analizar el currículo ecuatoriano para niños de subnivel Inicial II (cuatro años) y Preparatoria (cinco años) con un enfoque en patrones matemáticos, a la luz de la literatura internacional. Dicho análisis se centró en tres componentes: las destrezas planteadas en cada currículo, la secuencia propuesta para trabajar patrones matemáticos, y las estrategias recomendadas para la enseñanza y aprendizaje de patrones matemáticos. Se da inicio a esta esta última sección resaltando el hecho de que los currículos ecuatorianos de subnivel Inicial II y de Preparatoria propongan destrezas referentes a patrones matemáticos, pues dada la importancia de los patrones matemáticos, un número cada vez mayor de países han incluido este contenido en sus currículos para preescolar y jardín infantil (Wijns et al., 2019). A continuación, se presentan las principales conclusiones del estudio.

En general, aunque se considere a los patrones matemáticos como un contenido necesario a trabajar dentro de los currículos ecuatorianos, en el subnivel Inicial II existe solamente una destreza sobre patrones matemáticos y en Preparatoria solamente tres destrezas (dos de las cuales no son imprescindibles sino deseables). Además, se observó que los currículos ecuatorianos para los niveles estudiados incluyen principalmente el trabajo con patrones de repetición sencillos, lo cual concuerda con estudios previos que señalan que los patrones de repetición se consideran el tipo de patrón más accesible para los niños pequeños (Rittle-Johnson et al., 2013). Sin embargo, la literatura internacional señala que, sobre todo en el jardín de infantes (Preparatoria en nuestro contexto), el trabajo con patrones de crecimiento es también considerado importante ya que 
permite a los niños desarrollar una comprensión más profunda de la regla que subyace a un patrón (Papic y Mulligan, 2007; Rittle-Johnson et al., 2013), lo cual es fundamental para el desarrollo del pensamiento matemático. Si bien, el Ministerio de Educación (2016) señala que se da la apertura para que las instituciones y/o docentes puedan ampliar los contenidos prescritos en los documentos curriculares, de acuerdo a las necesidades particulares y al contexto, Lüken (2018) aclaró que es común que, en la enseñanza de niños pequeños, los profesores no usen definiciones explícitas y con frecuencia los patrones y las estructuras se manejen de manera implícita por lo que, sería conveniente incluir de manera explícita en el currículo nacional un mayor número de destrezas relacionadas con patrones matemáticos e incluir patrones de crecimiento para ser trabajados por los niños de cuatro y cinco años de edad.

Por otro lado, en los currículos analizados se evidenció la ausencia de una secuencia clara a seguir para el trabajo con patrones matemáticos, por ello se recomienda que dichos currículos incluyan pautas sobre las trayectorias del aprendizaje de patrones basadas en investigaciones previas y descritas en la literatura, con la finalidad de que los docentes conozcan dichas trayectorias, reflexionen sobre las mismas en base al conocimiento de sus estudiantes, y planifiquen sus clases teniendo en cuenta el desarrollo de aprendizaje natural de sus niños (ver por ejemplo las trayectorias de aprendizaje de patrones descritas en Clements y Sarama, 2014). A más de las trayectorias de aprendizaje sería importante incluir, explícitamente, en los documentos curriculares estrategias metodológicas que abarquen diferentes modalidades para trabajar con patrones matemáticos, por ejemplo, presentar actividades auditivas y táctiles como las descritas en la sección anterior, así como diferentes tipos de tareas, sobre todo tareas de identificar la unidad mínima del patrón, lo cual es fundamental para el desarrollo del pensamiento matemático del niño. El identificar la unidad mínima de patrón permitirá a los niños generalizar este aprendizaje a otros temas en los cuales los patrones están presentes, por ejemplo, a los principios del conteo y la aritmética que describen generalizaciones de secuencias predecibles, como ser: el siguiente número en la secuencia del conteo representa una magnitud que es exactamente uno más que el número anterior (la función sucesora; Rittle-Johnson et al., 2015).

Desde el punto de vista educativo, el presente estudio provee recomendaciones puntuales para revisar y optimar las destrezas, actividades, secuencias y estrategias, relacionadas con los patrones matemáticos prescritos en los documentos curriculares, con miras a mejorar las competencias, en patrones en particular y en matemáticas en general, de los niños ecuatorianos desde los primeros años de escolaridad. Finalmente, una limitación del presente estudio es que el análisis de los currículos se ha centrado exclusivamente en patrones matemáticos, dejando de lado los demás componentes de la matemática. Sería conveniente que futuros estudios realicen un análisis integral de todos los componentes de la matemática que abarcan los documentos curriculares nacionales. Tales estudios servirán para informar a los encargados de la política educativa y del diseño del currículo sobre el contenido, la secuencia y la metodología convenientes para promover una educación matemática adecuada en los niveles iniciales, que les permita a los niños desarrollar bases sólidas sobre las cuales construir aprendizajes futuros. 


\section{Referencias Bibliográficas}

Aunio, P., \& Niemivirta, M. (2010). Predicting children's mathematical perfomance in grade one by early numeracy. Learning and Individual Differences, 20, 427-435.

Autor. (2015).

Bailey, D., Nguyen, T., Jenkins, J. M., Domina, T., Clements, D. H., \& Sarama, J. (2016). Fadeout in an early mathematics intervention: Constraining content or preexisting differences? 52(9), 1457-1469. https://doi.apa.org/doiLanding?doi=10.1037\%2Fdev0000188. Obtenido de https://doi.org/10.1037/dev0000188

Baroody, A. (2004). The role of psychological research in the development of early childhood. En D. H. Clements, J. Sarama y A.-M. DiBiase (Eds.). Engaging young children in mathematics: Standards for early childhood mathematics education, 149-172.

Baroody, A., Clements, D., \& Sarama, J. (2019). Teaching and learning mathematics in early childhood programs. In C. Brown, M. McMullen, \&amp; N. K. File (Eds.). Handbook of early childhood care and education. (Section III of Curriculum in Early Childhood Care and Education). Hoboken, NJ: Wiley Blackwell Publishing.

Björklund, C., \& Pramling, N. (2014). Pattern discernment and pseudo-conceptual development in early chidhood matehematics education. 22, 89-104. Obtenido de International Jornal of Early Years Education: https://doi.org/10.1080/09669760.2013.809657

Clements, D. H., \& Sarama, J. (2014). Learning and teaching early math: The learning trajectories approach. 2. Nueva York, NY, Routledge.

Coburn, T., Bushey, B., Holton, L., Latozas, D., Mortimer, D., \& Shotwell, D. (1993). Patterns.Curriculum and evaluation standards for school mathematics. Addenda Series, Grades K-6. Reston, Va.: National Council of Teachers of Mathematics.

Duncan, G. J., Dowsett, C. J., Claessens, A., Magnuson, K., Huston, A. C., Klebanov, P., \& Japel, C. (2007). School readiness and later achievement. Developmental Psychology, 43, 14261446.

Economopoulos, K. (1998). What comes next? The mathematics of pattern in kindergarten. Teaching Children Mathematics, 5, 4, 230-233. Obtenido de http://www.jstor.org/stable/41197171

Epstein, Q. (2007). The Intentional Teacher: Choosing the Best Strategies for Young Children's. National Association for the Education of Young Children. Washington, DC:

Fyfe, E. R., Rittle-Johnson, B., \& DeCaro, M. S. (2012). The effects of feedback during exploratory mathematics problem solving: Prior knowledge matters. Journal of Educational Psychology, 104, 1094-1108.

Griffin, S. (2005). Fostering the development of number sense. Teaching mathematics in the early primary grades. En M. S. Donovan \&amp; J. D. Bransford (Eds.). How students learn: History, mathematics and science in the classrooms, 257-308. Washington, D. C.: The National Academies Press.

Jordan, N. C., Kaplan, D., Ramineni, C., \& Locuniak, M. N. (2009). Early math matters: Kindergarten number competence and later mathematics outcomes. Developmental Psychology, 45, 850-867.

Kidd, J. K., Carlson, A. G., Gadzichowski, M., Boyer, C. E., Allington, D., \& Pasnak, R. (2013). Effects of patterning instruction on the academic achievement of 1st-grade children. Journal of Research in Childhood Education, 27(2), 224-238. 
Kulm, G., \& Li, Y. (2009). Curriculum research to improve teaching and learning: National and cross-national studies. ZDM Mathematics Education, 41, 709-715.

Lüken, M. M. (2012). Young children's structure sense. Journal fur Mathematik-Didaktik. 33(2), 263-268. Obtenido de https://doi.org/10.1007/s13138-012-0036-8

Lüken, M. M. (2018). Repeating pattern competencies in three- to five-year old kindergartners: A closer look at strategies. Contemporary Research and Perspectives on Early Childhood Mathematics Education, 35-54. (Springer, Ed.) Hamburg: In C. B. Iliada Elia, Joanne Mulligan, Ann Anderson, Anna Baccaglini-Frank .

Lüken, M. M., Peter-Koop, A., \& Kollhoff, S. (2014). Influence of early repeating patterning ability on school mathematics learning. Proceedings of the 38th conference of the International Group for the Psychology of Mathematics Education and the 36th Conference of the North American Chapter of the Psychology of Mathematics education, In D. Allan, P. Liljedahl, C. Nicol, \&amp; S. Oesterle, 137-144. Vancouver, Canada.

Ministerio de Educación. (2014). Currículo Educación Inicial. Quito, Ecuador. Recuperado el 10 de enero de 2019, de https://educacion.gob.ec/wpcontent/uploads/downloads/2016/03/CURRICULO-DEEDUCACION-INICIAL.pdf

Ministerio de Educación. (2015). Guía metodológica para la implementación del currículo de educación inicial. Recuperado el 19 de enero de 2019, de https://educacion.gob.ec/wpcontent/uploads/downloads/2016/03/Guia-Metodologicapara-la-Implementacion-delCurriculo.pdf

Ministerio de Educación. (2016). Currículo de los niveles de educación obligatoria. Quito, Ecuador. Recuperado el 10 de enero de 2019, de https://educacion.gob.ec/wpcontent/uploads/downloads/2016/08/Curriculov2.pdf

Ministerio de Educación. (2017). Guía para el docente para Preparatoria de Educación General Básica. Quito, Ecuador. Recuperado el 10 de enero de 2019, de https://educacion.gob.ec/wpcontent/uploads/downloads/2018/04/curriculo/GuiaPreparatoria-1er-Grado.pdf

Ministerio de Educación. (2018). Relaciones lógico matemáticas. Preparatoria. Cuaderno de trabajo 2. Quito, Ecuador: Santillana. Recuperado el 10 de enero de 2019, de https://educacion.gob.ec/wpcontent/uploads/downloads/2018/08/RelacionesMatematicas-cuaderno-1ro-PREEGB.pdf

Mulligan, J., \& Mitchelmore, M. (2009). Awareness of Pattern and Structure in Early Mathematical Development. Mathematics Education Research Journal, 21(2), 33-49.

Nguyen, T., Watts, T. W., Duncan, G. J., Clements, D. H., Sarama, J. S., Wolfe, C., \& Spitler, M. E. (2016). Which preschool mathematicas competencies are most predictive of fifth grade achievement? Early Childhood Research Quaterly, 36, 550-560. Obtenido de https://doi.org/10.1016/j.ecresq.2016.02.003

Papic, M., \& Mulligan, J. (2007). Mathematical patterning in early childhood: an intervention study. Mathematics: Essential Research, Essential Practice, 2, 591-600. Recuperado el 10 de enero de 2019, de http://oatd.org/oatd/record?record=\%22handle:1959.14/215381\%22

Piccolo, D. L., \& Test, J. (2010). Preschoolers' thinking during block play. Teaching Children Mathematics, 17(5), 310-316. 
Rittle-Johnson, B., Fyfe, E. R., Hofer, K. G., \& Farran, D. C. (2017). Early math trayectories: Low-income children's mathematics knouledge from ages 4 to 11. Child Development, 88(5), 1727-1742. Obtenido de https://doi.org/10.1111/cdev.12662

Rittle-Johnson, B., Fyfe, E. R., Loehr, A. M., \& Miller, M. R. (2015). Beyond numeracy in preschool: Adding patterns to the equation. Early Childhood Research Quarterly, 31, 101112. Obtenido de https://doi.org/10.1016/j.ecresq.2015.01.005

Rittle-Johnson, B., Fyfe, E. R., McLean, L. E., \& McEldoon, K. L. (2013). Emerging understanding of patterning in 4-year-olds. Journal of Cognition and Development, 14(3), 376-396. Obtenido de https://doi.org/10.1080/15248372.2012.689897

Rittle-Johnson, B., Zippert, E. L., \& Boice, K. L. (2019). The roles of patterning and spatial skills in early mathematics development. Early Childhood Research Quaterly, 46, 166-178. Obtenido de https://doi.org/10.1016/j.ecresq.2018.03.006

Sarama, J., Clements, D. H., Starkey, P., Klein, A., \& Wakeley, A. (2008). Scaling up the implementation of a pre-Kindergarten mathematics curriculum: Teaching for understanding with trajectories and technologies. Journal of Research on Educational Effectiveness, 1, 89-119.

Schmidt, W. H., McKnight, C. C., Valverde, G. A., Houang, R. T., \& Wiley, D. E. (1997). Many Visions, Many Aims: A Cross-National Investigation of Curricular Intensions in School Mathematics. Dordrecht, The Netherlands: Kluwer.

Seo, K. H., \& Ginsburg, H. P. (2004). What is developmentally appropriate in early childhood mathematics education? lessons from new research. . In D. H. Clements, J. Sarama, \& A.M. DiBiase (Eds.), Engaging Young Children in Mathematics (pp. 91-104). Hillsdale,NJ: Erlbaum.

Starkey, P., Klein, A., \& Wakeley, A. (2004). Enhancing young children's mathematical knowledge through a pre-kindergarten mathematics intervention. Early Childhood Research Quarterly, 19, 99-120.

Stein, M., Remillard, J., \& Smith, M. (2007). How curriculum influences student learning. In F. K. Lester Jr. (Ed.), Second handbook of research on mathematics teaching and learning (pp. 319-369). Gweenwich, CT: Information Age.

Verschaffel, L., Torbeyns, J., \& De Smedt, B. (2017). Young children's early mathematical competencies: analysis and stimulation. Plenure Lecture Presented at the Congress of European Research in Mathematics Education (CERME). Dublin, Ireland.

Wijns, N., Torbeyns, J., De Smedt, B., \& Verschaffel, L. (2019). Young children's patterning competencies and mathematical development: A review. In K. Robinson, H. Osana, \& D. Kotsopoulos (Eds.). New York: Springer. 